\title{
The histone acetyltransferase GcnE regulates development and adherence in the human fungal pathogen Aspergillus fumigatus
}

Chi-Jan Lin and Ying-Lien Chen

Department of Plant Pathology and Microbiology, National Taiwan University, 10617 Taipei, Taiwan

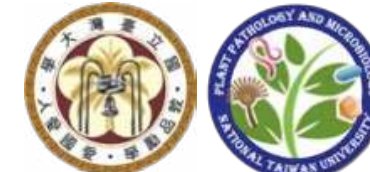

Histone modifications including acetylation, methylation and phosphorylation play a crucial role in gene regulations in eukaryotes. The acetylation module of SAGA type histone acetylation complex comprises of an acetyltransferase GcnE and two adaptor proteins AdaB and AdaC. In Aspergillus, GcnE has been shown to regulate asexual development and secondary metabolism in $A$. nidulans and $A$. flavus; however, knowledge of GcnE in the human pathogenic fungus $A$. fumigatus is limited. In this study, we showed that GcnE plays a role in conidiation and biofilm formation in $A$. fumigatus. We observed that the $\Delta g c n E$ mutant was unable to response to different nitrogen sources compared with the wild type. In addition, loss of gcnE resulted aberrant conidiation that conidiophores showed abnormal phialide formation. The $\Delta g c n E$ mutant exhibited increased susceptibility to cell wall perturbing agents and increased tolerance to $\mathrm{LiCl}$. Furthermore, we showed that GcnE is involved in biofilm formation and overexpression of adherence related genes (somA and uge3) partially rescued the defects of biofilm formation in the $\Delta g c n E$ mutant. Finally, GcnE is not involved in virulence in neutropenic murine model of invasive aspergillosis. Collectively, GcnE plays a crucial role in conidiation and biofilm formation but not in virulence in $A$. fumigatus.

1. GcnE is involved in nitrogen assimilation
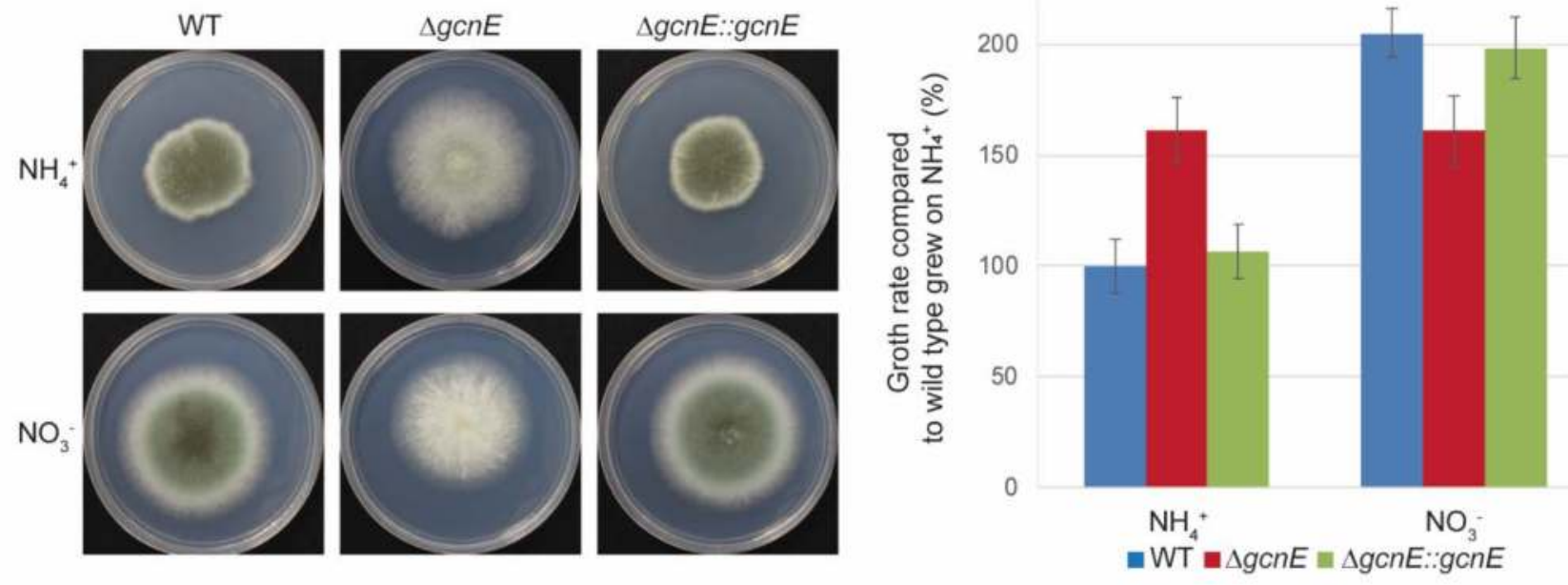

2. GcnE controls conidiation

WT

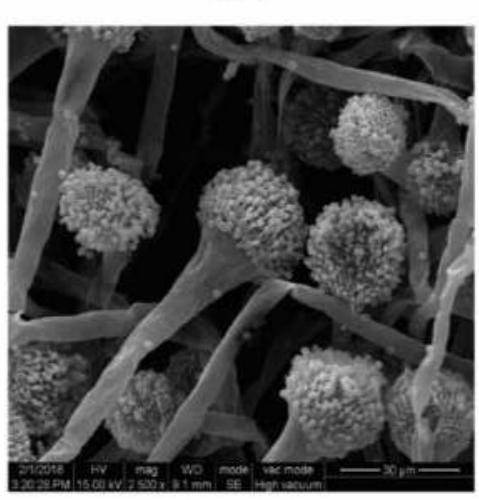

$\mathrm{NO}_{3}$

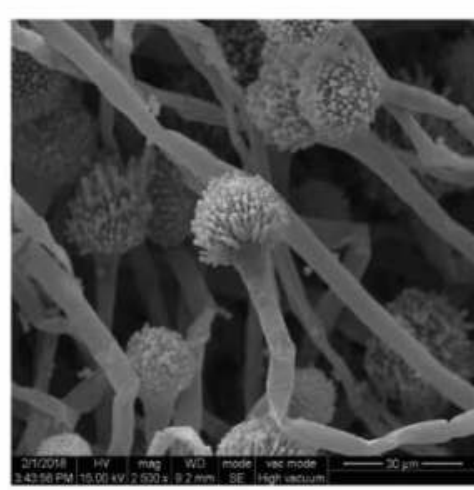

$\Delta g c n E$
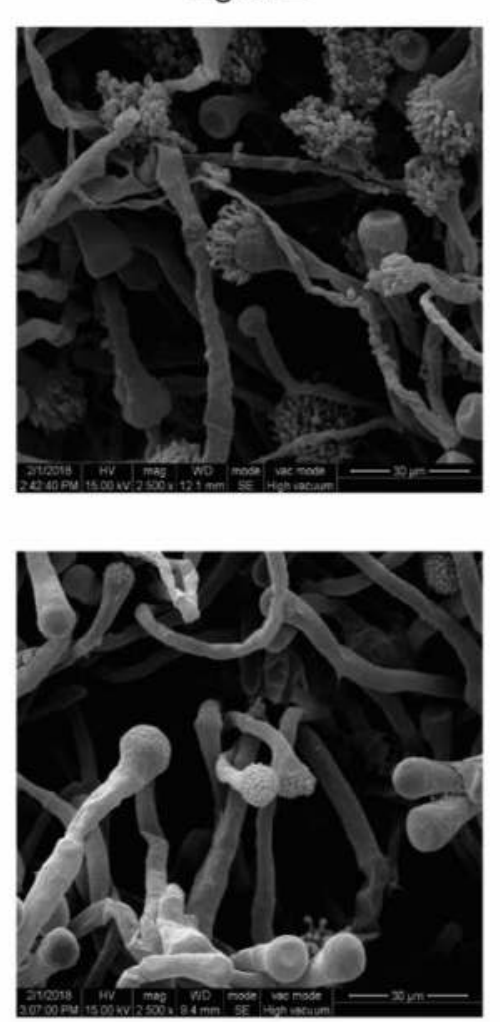

$\Delta g c n E:: g c n E$
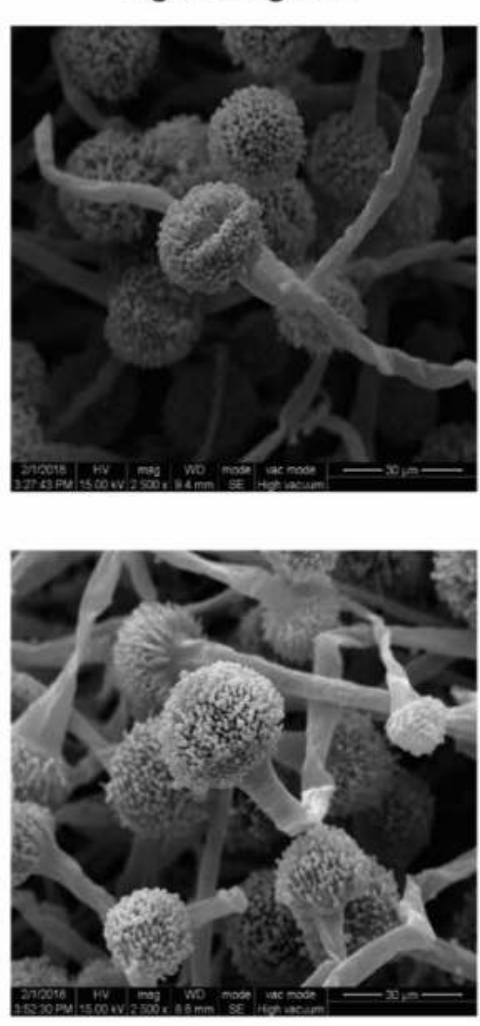

3. GcnE is involved in stress responses

MM

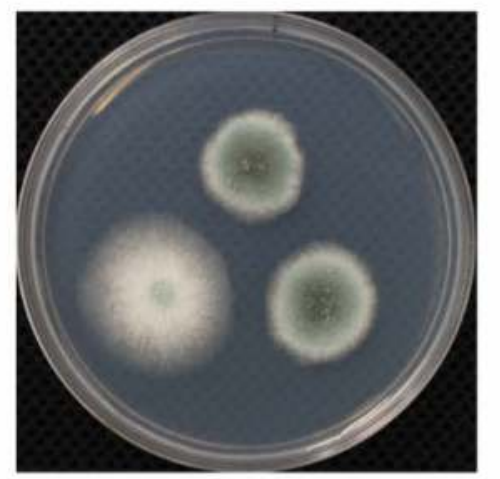

$\mathrm{H}_{2} \mathrm{O}_{2}(3 \mathrm{mM})$

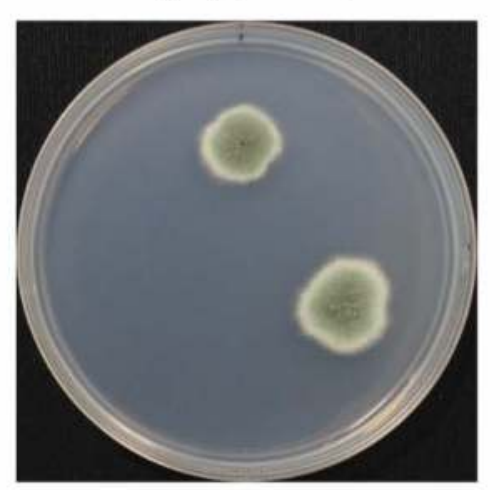

Congo Red $(30 \mu \mathrm{g} / \mathrm{mL})$

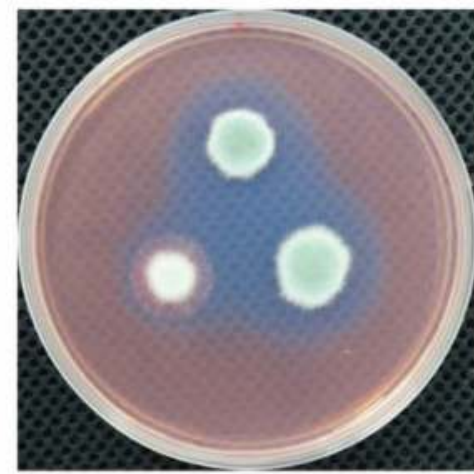

$\mathrm{LiCl}(100 \mathrm{mM})$

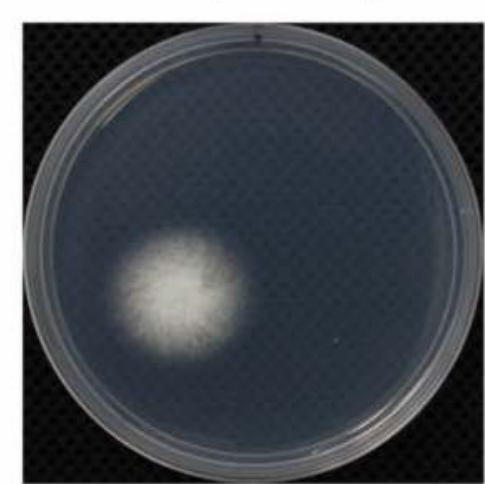

$\operatorname{SDS}(0.01 \%)$
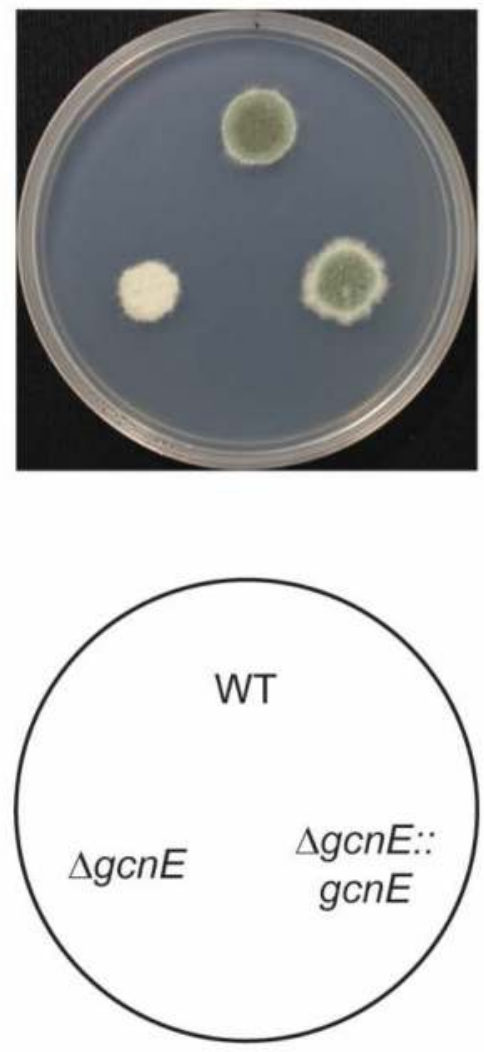

4. GcnE gorverns biofilm formation

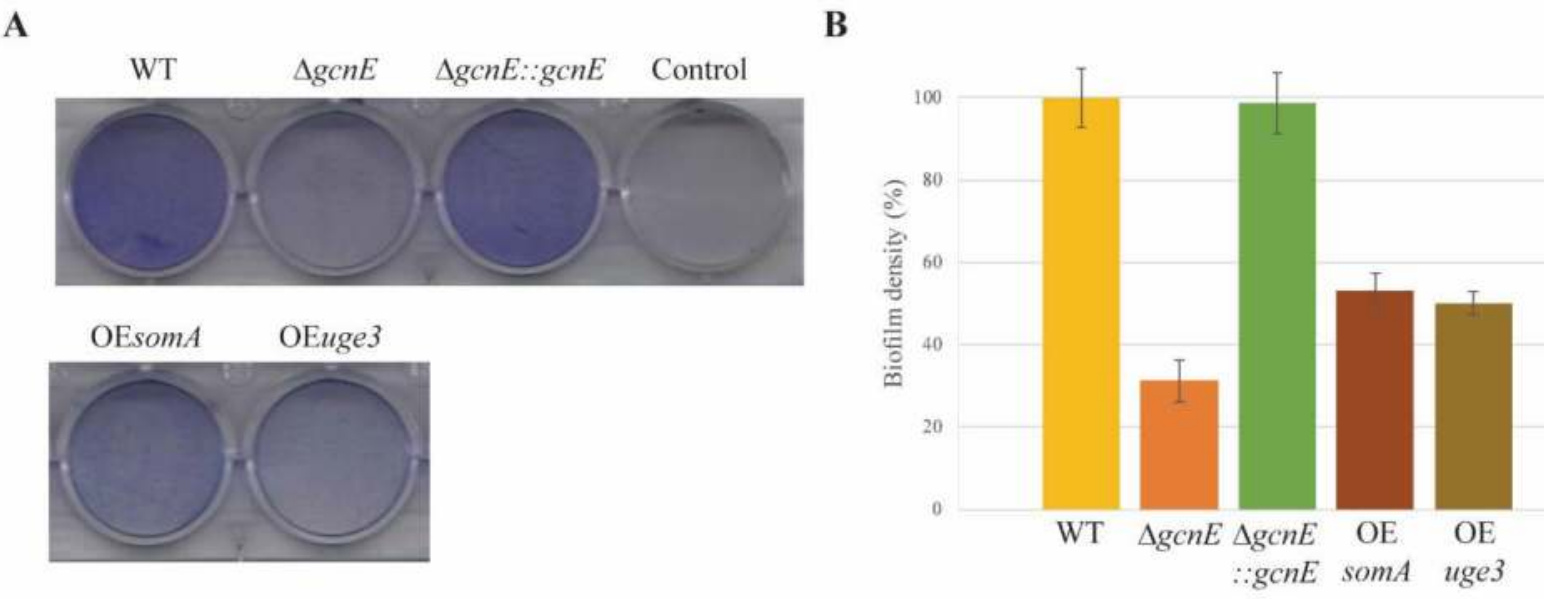

5. GcnE regulates gene for conidiation, adherence and ammonium transportation

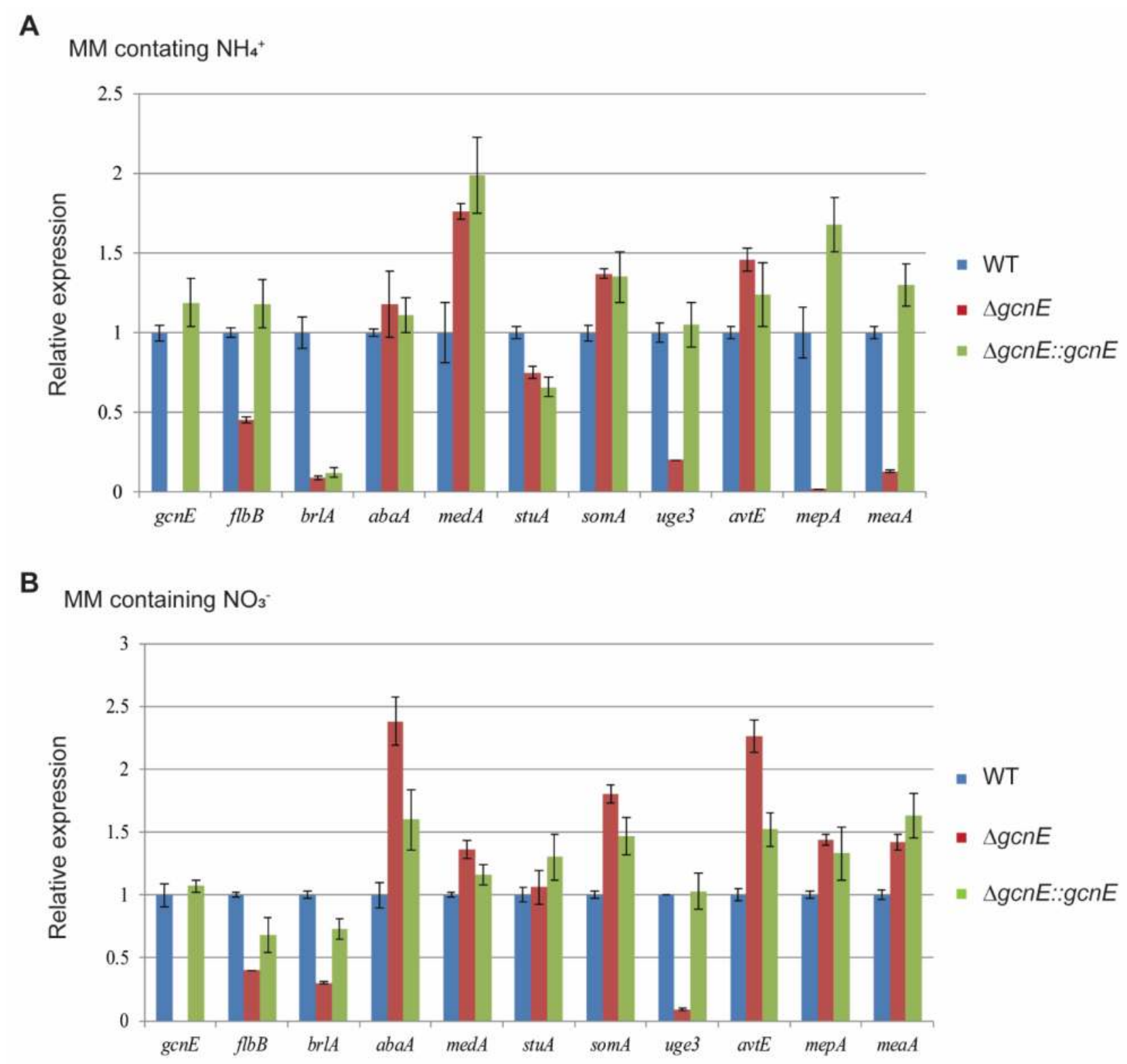

6. Virulence is independent of GcnE

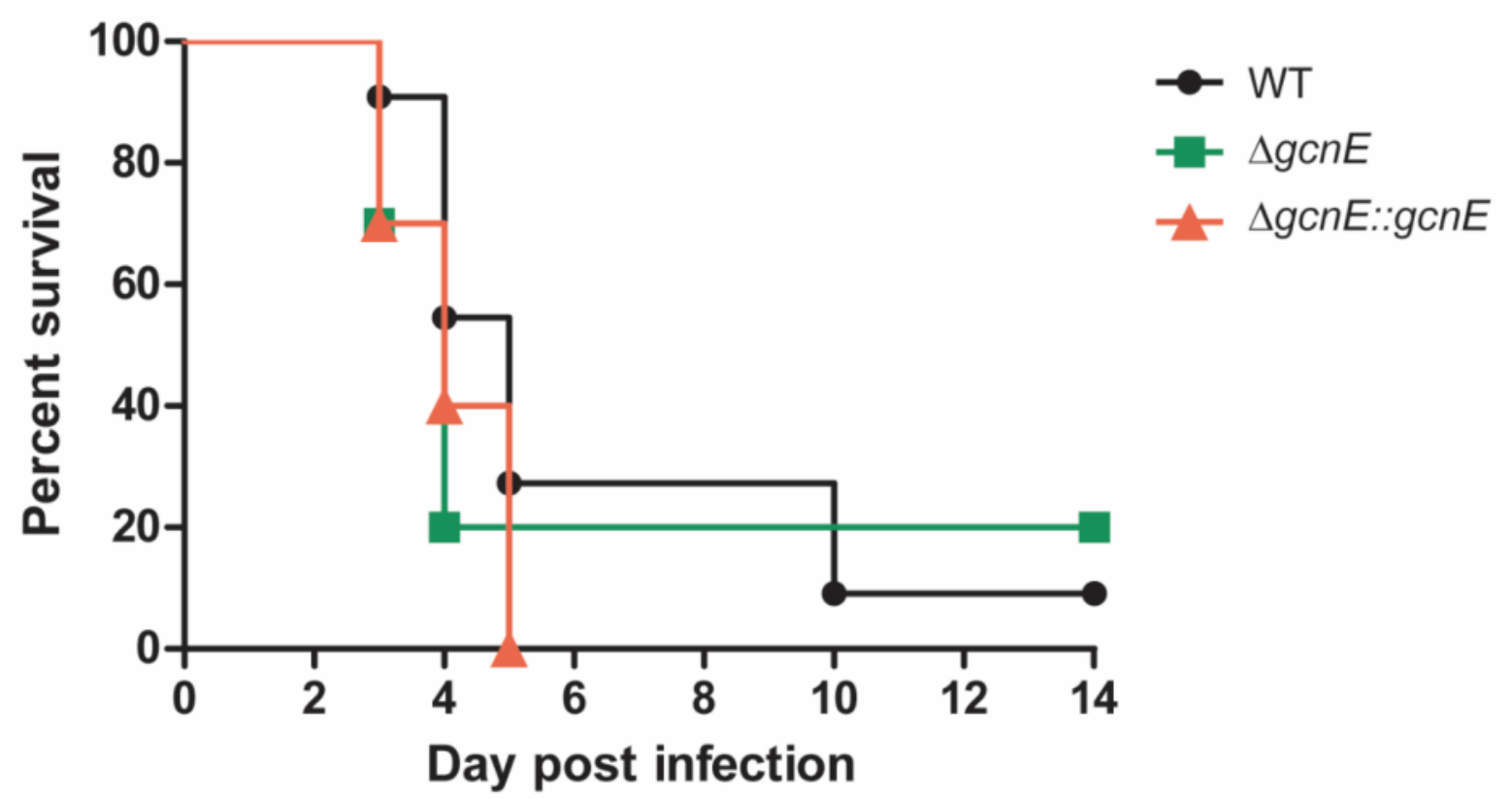

